\title{
Faixa Geotermal Anômala decorrente da "Colagem Tectônica" fraca na Margem Continental Brasileira
}

Fábio P. Vieira e Valiya M. Hamza, Observatório Nacional - ON/MCT, Rio de Janeiro

Copyright 2014, SBGf - Sociedade Brasileira de Geofísica

Este texto foi preparado para a apresentação no VI Simpósio Brasileiro de Geofísica, Porto Alegre, 14 a 16 de outubro de 2014. Seu conteúdo foi revisado pelo Comitê Técnico do VI SimBGf, mas não necessariamente representa a opinião da SBGf ou de seus associados. É proibida a reprodução total ou parcial deste material para propósitos comerciais sem prévia autorização da SBGt.

\section{Abstract}

Results of an updated analysis of geothermal data for sedimentary basins in the Brazilian continental margin (MCB) are presented. The analysis also includes updated data sets compiled recently for the adjacent oceanic and continental segments. The results obtained have allowed identification of an anomalous geothermal belt along the entire extent of the continental margin, characterized by systematically high temperature gradient and heat flow values relative to those of the neighboring areas adjacent to MCB. The width of anomalous belt is larger in the basins of the Southern region (Pelotas, Santos, Campos), when compared with those in the North (South Bahia) and Northeast (Potiguar and Ceará). Most of the off-shore oil and gas fields are located in the central parts of this belt, pointing to the existence of a direct association between the geothermal field and zones of hydrocarbon reservoirs. There are indications that the origin of this anomalous thermal belt is related to recent episodes of magma emplacement at shallow depths in the upper crust. We advent the hypothesis that such magmatic events arise from space-time variations in tectonic forces (such as ridge push), which control the bonding between continental and oceanic segments of the South American plate.

\section{Introdução}

A margem continental brasileira, que se estende por mais de $8.000 \mathrm{~km}$, é caracterizada por um sistema contínuo de bacias sedimentares. As características geológicas e geofísicas dessas bacias foram avaliadas em diversos estudos realizados nas últimas décadas (e.g. Asmus e Ponte, 1973; Cainelli e Mohriak, 1999) A maioria desses estudos se baseiam nos resultados de perfurações exploratórias realizados pela PETROBRÁS. Contudo, não foram efetuadas ainda análises detalhadas de dados de temperaturas adquiridos em poços de exploração. A falta de estudos detalhados de dados geotérmicos tem-se tornado um obstáculo no entendimento das características termo-tectônicas dos processos de distensão litosférica, atuantes na margem continental. Neste contexto o Laboratório de Geotermia do Observatório Nacional - ON/MCTI iniciou um projeto visando reavaliação dos dados geotérmicos da margem continental. Apresentam-se neste trabalho os resultados obtidos na fase inicial deste projeto, onde se destaca a descoberta de uma faixa geotermal anômala, confinado entre segmentos da crosta oceânica e continental da placa litosférica sul-americana.

\section{Contexto Tectônico}

A origem da MCB teve início no período Cretáceo, quando ocorreu a separação continental entre as placas litosféricas da África e da América do Sul. A abertura do oceano Atlântico foi acompanhado e sucedido por inúmeros eventos magmáticos e tectônicos e contribuiu para formação de diversas bacias na margem continental. Análises das características geológicas apontam atuação de mecanismos de distensão litosférica ao longo da margem entre a bacia de Pelotas e o extremo nordeste (Ojeda, 1982; Zalán, 1985; Szatmari et al., 1987; Mascle et al., 1988; Matos, 2000; Milani \& Thomaz Filho, 2000). Uma das características marcantes das bacias da MCB é ocorrência de episódios de atividades magmáticas e vulcânicas após o período inicial da separação continental. A margem continental brasileira pode ser dividida em diversos domínios tectônicos, englobando o segmento transformante da margem equatorial, o segmento transversal (passagem da margem equatorial para a margem nordeste) e os segmentos divergentes das margens nordeste, leste, sudeste e sul, cada qual com características estratigráficas e estruturais distintas (Cainelli e Mohriak, 1999). Além das bacias oceânicas, ocorrem também alguns riftes abortados que são parte integrante da evolução tectono-sedimentar da margem continental, estando relacionados à ruptura do Gondwana.

\section{Bases de Dados Utilizados}

Os dados geotérmicos utilizados neste trabalho foram baseados em grande parte nos trabalhos realizados por Ross e Pantoja (1978), Rossi Filho (1981), Zembruscki (1982), Del Rey e Zembruscki (1991), Viana (1999), Cardoso e Hamza (2003), entre outros. Recentemente esses dados foram reavaliados por Vieira e Hamza (2011) como parte de banco de dados geotérmicos globais. Também foram avaliados para finalidades deste trabalho dados de temperaturas do fundo do mar nas áreas da MCB, adquiridos pela Diretoria de Hidrografia e Navegação da Marinha Brasileira-DHN.

Os estudos realizados englobam dados geotérmicos de 696 poços na MCB. Desse conjunto 63 estão situados na área das bacias do Ceará e Potiguar, 121 na área das Bacias de Alagoas e Sergipe, 61 na área das bacias do Recôncavo, Camamu, Almada, Jequitinhonha e Cumuruxatiba, 319 na área da bacia do Espírito Santo e 132 na área das bacias de Campos, Santos e Pelotas. Foram selecionados apenas dados que permitiram determinações confiáveis de temperaturas em poços. $O$ mapa da figura (1) ilustra locais de dados avaliados no presente trabalho, onde os pontos indicam poços nos 
quais foram efetuadas medidas geotérmicas e as linhas tracejadas indicam os limites marítimos das bacias.

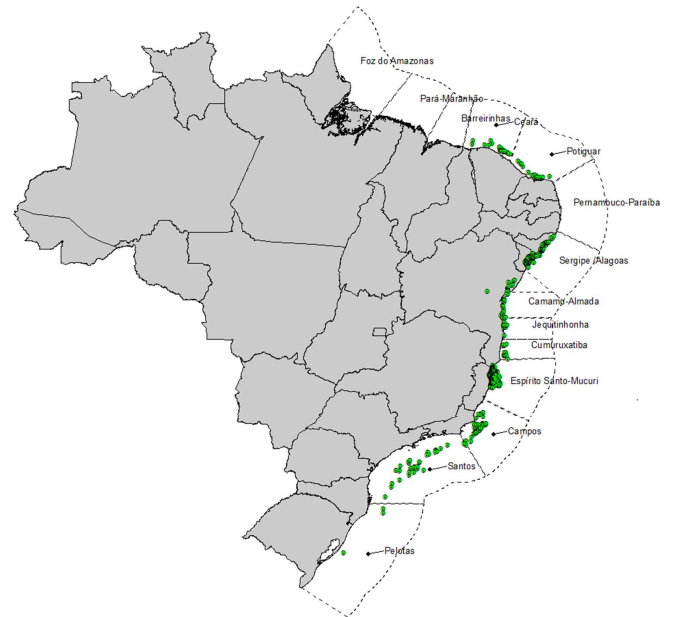

Figura 1 - Mapa indicando locais de poços selecionados (pontos de cor verde) nas bacias da MCB.

\section{Gradientes Geotérmicos}

Os dados de temperaturas de poços (BHT) foram corrigidos dos efeitos perturbadores de perfuração através do procedimento AAPG (1976). Esta correção é baseada no uso da relação:

$$
\Delta T=a \cdot z+b \cdot z^{2}-c \cdot z^{3}-d . z^{4}
$$

onde $\Delta T$ é a correção da temperatura, $z$ é a profundidade e $a, b, c, d$ são os coeficientes polinomiais com valores: $1.878 \times 10^{-3}, 8.476 \times 10^{-7}, 5.091 \times 10^{-11}$ e $1.681 \times 10^{-14}$ respectivamente.

As temperaturas do fundo do poço $\left(\mathrm{T}_{\mathrm{BH}}\right)$ na profundidade $Z_{B H}$ e fundo do mar $\left(T_{F M}\right)$ na profundidade $Z_{F M}$ foram utilizados nas determinações de gradientes geotérmicos (Г), com base na relação:

$$
\Gamma=\frac{T_{B H}-T_{F M}}{Z_{B H}-Z_{F M}}
$$

Nas determinações da temperatura do fundo do mar utilizou-se a relação proposta por Cardoso e Hamza (2003). Constam na Tabela (1) os valores médios de gradientes geotérmicos das bacias.

Table 1. Valores médios de gradientes geotérmicos $(\Gamma)$ das bacias na MCB.

\begin{tabular}{|l|c|c|c|}
\hline Bacia / Região & $\mathbf{N}$ (dados) & $\boldsymbol{\Gamma}$ & $\boldsymbol{\sigma}_{\mathbf{g}}$ \\
\hline Ceará & 37 & 36.3 & 4.0 \\
\hline Potiguar & 26 & 39.9 & 3.4 \\
\hline Alagoas & 44 & 24.6 & 3.9 \\
\hline Sergipe & 77 & 27.7 & 5.4 \\
\hline Reconcavo & 12 & 26.2 & 3.0 \\
\hline Bahia Sul & 49 & 58.3 & 14.5 \\
\hline Campos & 76 & 30.3 & 2.7 \\
\hline Estado do RJ & 22 & 16.7 & 4.1 \\
\hline Santos & 31 & 31.0 & 3.4 \\
\hline Pelotas & 3 & 31.7 & 2.3 \\
\hline
\end{tabular}

\section{Condutividade Térmica}

Medições experimentais de condutividade térmica foram realizadas em amostras da bacia do Recôncavo (Carvalho, 1981), de Sergipe-Alagoas (Andrade Fontes, 1980) e Espirito Santo e Mucuri (Del Rey and Zembruscki, 1991). Em 33 poços das bacias de Campos e de Santos os valores de condutividade térmica foram determinados com base na descrição litológica de amostras coletadas na perfuração (Viana, 1999; Cardoso e Hamza, 2003). Nas demais bacias utilizou-se o método proposto por Houbolt e Wells (1980) para estimar a condutividade térmica a partir de perfis sônicos.

De modo geral os valores de condutividade térmica apresentaram variações relacionadas com tipo litológico, porosidade e profundidade. Constatou que as formações ricas em folhelho se encontram caracterizadas por valores relativamente baixos de condutividade térmica em relação aos com teores elevados de arenitos. Apresentam-se na Tabela (2) os valores médios representativos de condutividade térmica das principais formações sedimentares nas bacias na MCB.

\section{Fluxo Geotérmico}

Os valores de fluxo geotérmico foram determinados como produto de valores representativos de condutividade térmica e gradiente geotérmico. Utilizou-se a seguinte relação entre as temperaturas do fundo de poço $\left(\mathrm{T}_{\mathrm{BH}}\right)$ e do fundo do mar $\left(T_{F M}\right)$ e a condutividade térmica utilizada nas determinações de fluxo geotérmico (Cardoso e Hamza, 2014):

$$
q=\left(T_{B H}-T_{F M}\right) / \sum_{i=1}^{N} R_{i} Z_{i}
$$

No qual $N$ é o numero de camadas, $R_{i}$ a resistividade térmica (inverso de condutividade térmica) da camada $i$ com espessura $Z_{i}$. A somatória sobre as $N$ camadas permite determinação da resistênica térmica cumulativa até a profundidade de medida de BHT. Segue na Tabela (3) uma síntese dos valores médios de fluxo geotérmico nas principais bacias da MCB.

\section{Campo Geotermal das Áreas Adjacentes}

Os dados BHT de poços revelaram variações significativas nos gradientes geotermais na MCB. A compreensão da natureza dessas variações exige análises integradas do campo geotérmico das regiões adjacentes. Neste contexto, foram reunidos os dados geotérmicos das áreas continentais e oceânicas próximos da margem continental. A distribuição de dados de fluxo geotérmico, extraído da compilação recente de Vieira e Hamza (2011), é ilustrado na Figura (2). Nas determinações de valores representativos os dados foram reunidos em células de $1^{\circ} \times 1^{\circ}$. Foram selecionadas 46 células nas áreas continentais e 49 células nas áreas oceânicas. Os locais dessas células se encontram indicados no mapa da Figura (2).

Nota-se que a MCB se encontra limitada pelos segmentos da crosta continental (maior parte de idade Pré-cambriana) no lado oeste e pelos segmentos da crosta oceânica no lado leste. Assim os valores representativos de gradientes e fluxo geotérmico das células foram selecionados com base nos respectivos 
valores desses segmentos crustais. Como exemplo ilustrativo apresenta-se nas Tabelas (4) e (5) os valores de gradientes e fluxo térmico das células selecionadas.

Tabela (2) Valores de condutividade térmica $(\lambda)$ das principais formações sedimentares na MCB.

\begin{tabular}{|c|c|c|}
\hline Bacia & Formação / Campo & Intervalo de $\lambda$ \\
\hline \multirow{5}{*}{ Santos e Pelotas } & Marambaia & $1,6-2,6$ \\
\hline & Santos & $1,8-2,8$ \\
\hline & Itajaí-Açu & $1,7-2,9$ \\
\hline & Guarujá & $1,6-2,0$ \\
\hline & Ariri & $2,4-2,8$ \\
\hline \multirow{6}{*}{ Campos } & Emborê & $2,2-2,7$ \\
\hline & Grussai e Siri & $1,9-2,2$ \\
\hline & Macaé & $2,4-3,2$ \\
\hline & Ubatuba & $2,0-2,8$ \\
\hline & Carpebus & $2,0-3,0$ \\
\hline & Lagoa Feia & $1,8-2,4$ \\
\hline \multirow{5}{*}{$\begin{array}{l}\text { Espirito Santo e } \\
\text { Mucuri }\end{array}$} & Rio Doce & $1,6-1,9$ \\
\hline & Caravelas & 2,3 \\
\hline & Urucutuca & $1.9-2,7$ \\
\hline & Barra Nova & $1.7-3,0$ \\
\hline & Mariricu & $2.4-5,7$ \\
\hline \multirow{7}{*}{ Bahia Sul } & Rio Doce & $1,6-2,0$ \\
\hline & Algodões & $2,5-2,7$ \\
\hline & Taipu Mirim & $2,5-2,7$ \\
\hline & Rio de Contas & $2,4-2,8$ \\
\hline & Morro do Barro & $2.1-2,3$ \\
\hline & Itaípe & $2,2-2,5$ \\
\hline & Sergi & $3,2-3,6$ \\
\hline \multirow{6}{*}{ Recôncavo } & Araças & $2,2-2,6$ \\
\hline & Água Grande & $2,2-2,6$ \\
\hline & Buracica & $2,2-2,6$ \\
\hline & Fazenda Imbé & $2,2-2,6$ \\
\hline & Miranga & $2,2-2,6$ \\
\hline & Taquipe & $2,2-2,6$ \\
\hline \multirow{12}{*}{ Sergipe / Alagoas } & Piaçabuçu & $1,6-1,8$ \\
\hline & Cotinguiba & $2,0-2,4$ \\
\hline & Riachuelo & $1,6-1,8$ \\
\hline & Muribeca & $1,7-2,1$ \\
\hline & Coqueiro Seco & $1,6-1,8$ \\
\hline & Morro Chaves & $2,2-2,6$ \\
\hline & Rio Pitanga & $2,7-3,1$ \\
\hline & Penedo & $2,3-2,7$ \\
\hline & Barra Itiuba & $2,3-2,7$ \\
\hline & Serraria & $2,7-3,1$ \\
\hline & Bananeiras & $2,1-2,5$ \\
\hline & Batinga & $2,3-2,7$ \\
\hline \multirow{10}{*}{ Potiguar / Ceará } & Tibau & $2,3-2,5$ \\
\hline & Guamaré & $2,3-2,5$ \\
\hline & Ubarana & $2,3-2,5$ \\
\hline & Paracurú & $2,3-2,5$ \\
\hline & Mandaú & $2,3-2,5$ \\
\hline & Jandaíra & $2,3-2,5$ \\
\hline & Quebradas & $2,3-2,5$ \\
\hline & Alagamar & $2,3-2,5$ \\
\hline & Pescada & $2,3-2,5$ \\
\hline & Pendência & $2,3-2,5$ \\
\hline
\end{tabular}

Tabela (3) Valores médios de fluxo térmico (q) nas bacias de MCB.

\begin{tabular}{|c|c|c|}
\hline Bacia / Área & $\mathbf{q}\left(\mathbf{m W} / \mathbf{m}^{2}\right)$ & $\boldsymbol{\sigma}_{\mathrm{q}}\left(\mathbf{m W} / \mathbf{m}^{2}\right)$ \\
\hline Ceará e Potiguar & 94 & 9 \\
\hline Alagoas e Sergipe & 56 & 10 \\
\hline Recôncavo & 67 & 10 \\
\hline $\begin{array}{c}\text { Camamu, Almada, } \\
\text { Jequitionha e Cumuruxatiba }\end{array}$ & 103 & 35 \\
\hline Espírito Santo e Mucuri & 71 & 7 \\
\hline Campos & 73 & 10 \\
\hline Santos e Pelotas & 70 & 9 \\
\hline
\end{tabular}

Nas determinações de valores representativos de gradiente e fluxo geotérmico dessas células foram utilizados resultados de medições experimentais e valores estimados. As células de cor azul e verde indicam presença de dados observacionais enquanto as de cor amarela indicam ausência dos mesmos. A fim de obter uma base homogeneizada de dados foram estimados valores de gradientes e fluxo geotérmico para as células de cor amarela com base na relação empirica entre idade tectônica e fluxo geotérmico proposto por Polyak e Smirnov (1968) e Hamza e Verma (1969). As idades das áreas continentais e oceânicas adjacentes à MCB são ilustradas no mapa da Figura (3).

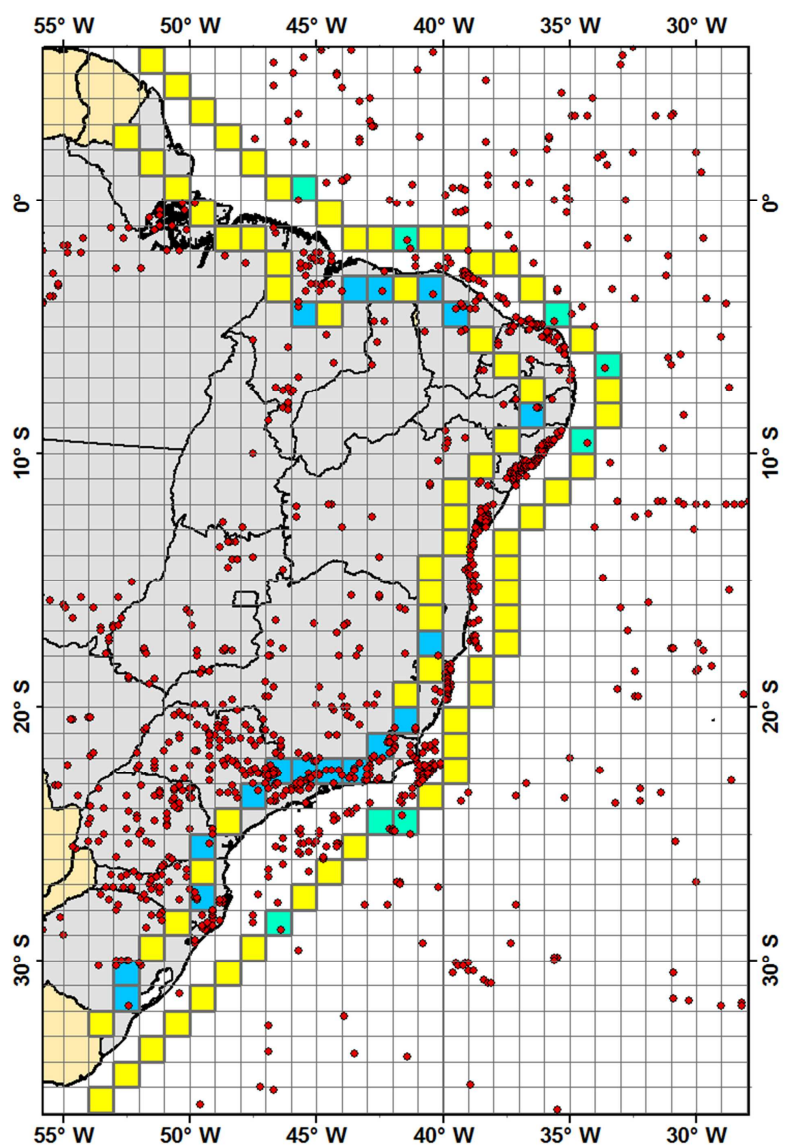

Figura 2 - Sistema de células usado para as áreas continentais e oceânicas adjacentes da MCB. 


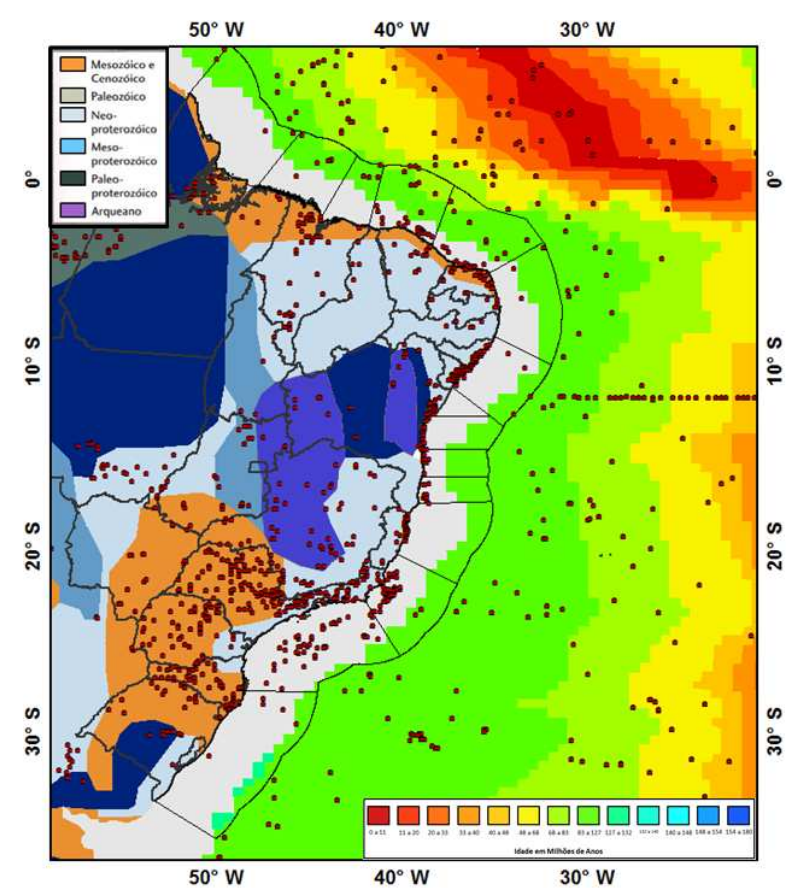

Figura 3 - Idades das áreas continentais e oceânicas adjacentes da MCB.

Tabela (4) valores representativos de gradientes ( $Г$ ) e de fluxo térmico (q) das células na área continental.

\begin{tabular}{|c|c|c|c|}
\hline Células & $\begin{array}{c}\Gamma \\
\left({ }^{0} \mathrm{C} / \mathrm{km}\right) \\
\end{array}$ & $\underset{\left(m W / m^{2}\right)}{q}$ & $\begin{array}{c}\text { Idade } \\
\text { Tectônica } \\
\end{array}$ \\
\hline 1 e 2 & 15 & 36 & $\begin{array}{c}\text { Paleo- } \\
\text { Proterozóico }\end{array}$ \\
\hline $\begin{array}{c}3,4,5,6,7,8,42 \\
\text { e } 43\end{array}$ & 25 & 54 & $\begin{array}{l}\text { Mesozóico/ } \\
\text { Cenozóico }\end{array}$ \\
\hline 9 & 26 & 54 & $\begin{array}{c}\text { Pré- } \\
\text { Cambriano }\end{array}$ \\
\hline $\begin{array}{c}10,13,16,17,18, \\
20,21,26,27,29, \\
30,38,40 \text { e } 46\end{array}$ & 18 & 43 & $\begin{array}{c}\text { Neo- } \\
\text { Proterozóico }\end{array}$ \\
\hline 11 & 20 & 48 & \multirow{5}{*}{$\begin{array}{c}\text { Pré- } \\
\text { Cambriano }\end{array}$} \\
\hline 12 & 20 & 48 & \\
\hline 14 & 20 & 48 & \\
\hline 15 & 20 & 48 & \\
\hline 19 & 20 & 48 & \\
\hline $22,23,24,25$ & 15 & 36 & Arqueano \\
\hline 28 & 19 & 46 & \multirow{6}{*}{$\begin{array}{c}\text { Pré- } \\
\text { Cambriano }\end{array}$} \\
\hline 31 & 19 & 46 & \\
\hline 32 & 20 & 48 & \\
\hline 33 & 19 & 46 & \\
\hline 34 & 19 & 46 & \\
\hline 35 & 19 & 46 & \\
\hline 36 & 19 & 46 & \multirow{2}{*}{ Fanerozóico } \\
\hline 37 & 19 & 46 & \\
\hline 39 & 23 & 55 & Précambriano \\
\hline 41 & 19 & 46 & Fanerozóico \\
\hline 44 & 19 & 46 & \multirow{2}{*}{$\begin{array}{c}\text { Pré- } \\
\text { Cambriano }\end{array}$} \\
\hline 45 & 19 & 46 & \\
\hline
\end{tabular}

Tabela (5) Valores representativos de gradiente $(\ulcorner)$ e de fluxo térmico (q) das células na área oceânica.

\begin{tabular}{|c|c|c|c|}
\hline Células & $\begin{array}{c}\mathbf{r} \\
\left({ }^{\mathbf{C} / \mathbf{k m})}\right)\end{array}$ & $\begin{array}{c}\mathbf{q} \\
\left(\mathbf{m W} / \mathbf{m}^{2}\right)\end{array}$ & $\begin{array}{c}\text { Idade } \\
(\mathbf{M a})\end{array}$ \\
\hline $\begin{array}{c}1,2,3,4,5,6,8,9,10,12, \\
13,14,15,16,18,20,21,23,\end{array}$ & & & \\
$\begin{array}{c}24,25,26,27,28,29,30,31, \\
32,33,34,35,36,39,40,41, \\
43,44,45,46,47,48 \text { e 49 }\end{array}$ & 22 & 53 & $>90$ \\
\hline 7 & 18 & 43 & $>120$ \\
\hline 11 & 22 & 53 & $80-120$ \\
\hline 17 & 22 & 53 & $80-120$ \\
\hline 19 & 18 & 43 & $80-120$ \\
\hline 22 & 28 & 54 & $80-120$ \\
\hline 37 & 24 & 52 & $80-120$ \\
\hline 38 & 29 & 52 & $80-120$ \\
\hline 42 & 21 & 50 & $80-120$ \\
\hline
\end{tabular}

\section{Variações Regionais no Campo Geotérmico}

Os resultados obtidos no presente trabalho indicam valores médios de gradientes geotérmicos na faixa de 25 à $59^{\circ} \mathrm{C} / \mathrm{km}$ (Tabela 1) para as bacias da MCB. No entanto, a maior parte dos valores de gradientes dos segmentos crustais das áreas adjacentes das bacias são significativamente menores, estando na faixa de 15 à $29^{\circ} \mathrm{C} / \mathrm{km}$ (Tabelas 4 e 5 ). Da mesma forma, os valores médios de fluxo geotérmico das bacias de MCB estão na faixa de 56 à $103 \mathrm{~mW} / \mathrm{m}^{2}$ (Tabela 3 ) enquanto que nas áreas adjacentes da MCB os valores de fluxo térmico são significativamente menores, estando na faixa de 36 à $54 \mathrm{~mW} / \mathrm{m}^{2}$. Esses contrastes no campo geotérmico entre as bacias consideradas e as suas áreas adjacentes implica na existência de uma faixa geotermal anômala na MCB.

A fim de ilustrar a natureza dessa faixa geotermal anômala foram elaborados mapas geotermais em escala regional. O mapa apresentado na Figura (4) indica ocorrências de gradientes geotérmicos com valores no intervalo de 25 à $59^{\circ} \mathrm{C} / \mathrm{km}$ nas partes centrais das bacias de MCB. Por outro lado, as áreas vizinhas de MCB, tanto na parte continental como na área oceânica, se encontram caracterizados por gradientes geotérmicos na faixa de 15 à $29^{\circ} \mathrm{C} / \mathrm{km}$. A figura (4) também aponta as variações na largura da faixa geotermal anômala. Nas regiões Sul e Sudeste (abrangendo as bacias de Pelotas, Santos e Campos) as larguras são relativamente maiores, alcançando valores de até $300 \mathrm{~km}$. Nas regiões do Nordeste e Norte as larguras da faixa geotermal não ultrapassam $200 \mathrm{~km}$.

O mapa da Figura (5) indica variações regionais no fluxo geotérmico na MCB e áreas vizinhas. Nota-se que as partes centrais das bacias da MCB são caracterizadas por fluxo geotérmico com valores na faixa de 56 à $103 \mathrm{~mW} / \mathrm{m}^{2}$. Por outro lado as áreas vizinhas nas partes continentais e oceânicas os valores de fluxo geotérmico são significativamente menores, na faixa de 36 à $54 \mathrm{~mW} / \mathrm{m}^{2}$. A figura (5) também aponta as variações na largura da faixa geotermal anômala, semelhantes àquelas observadas no mapa de gradientes geotérmicos. 


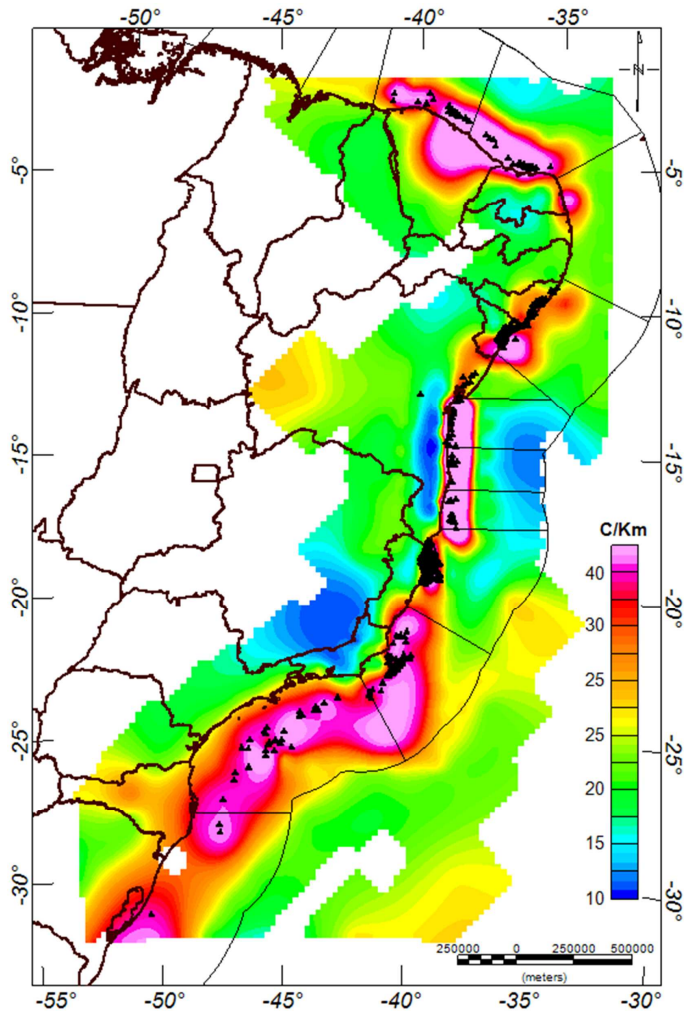

Figura 4 - Mapa illstrando variações nos gradientes geotérmicos ao longo da MCB.

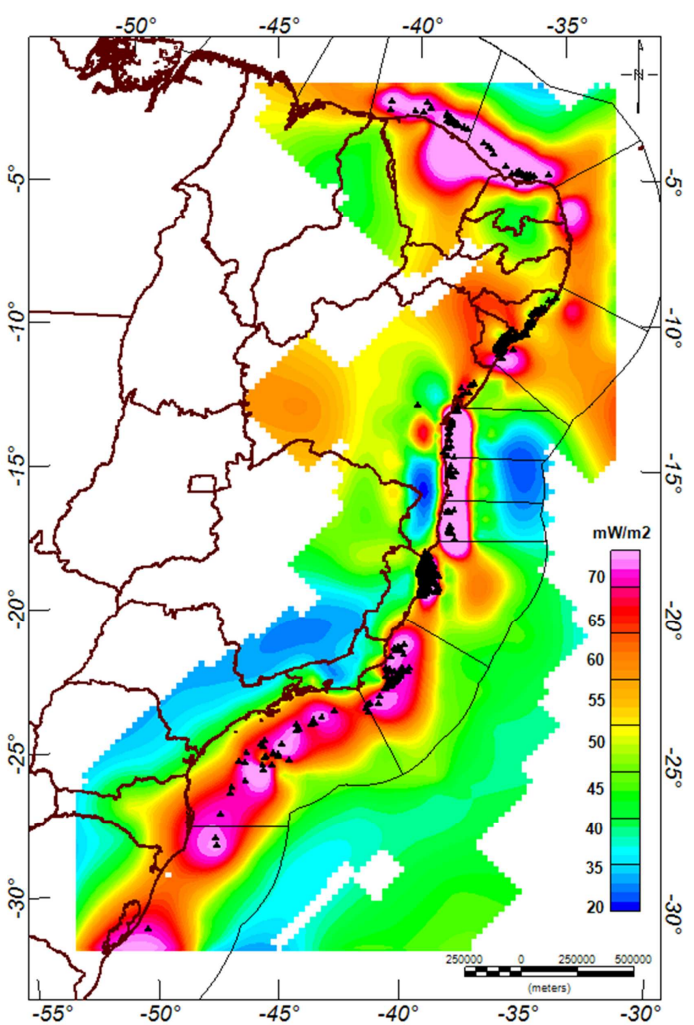

Figura 5 - Mapa ilustrando variações no fluxo geotérmico ao longo da MCB.

\section{Discussão e Conclusões}

A origem da faixa geotermal anômala parece não está vinculada aos processos de separação entre África e América do Sul, pois não foram observadas variações sistemáticas (na direção norte-sul) nos gradientes e fluxo geotérmico na MCB. Por outro lado, as características das variações regionais no campo térmico parecem estar em boa concordância com efeitos térmicos esperados das atividades magmáticas recentes ao longo da MCB. Aventa-se neste contexto a hipótese de enfraquecimento na "colagem tectônica" entre os segmentos continental e oceânico da placa Sul-americana na MCB. [Nota-se: A terminologia utilizada no presente trabalho ("colagem tectônica") é diferente daquela utilizada em geologia para descrever processos semelhantes nas atividades orogênicas ("colagem orogênica")]. Atenuação das forças (tipo "ridge-push") que garantem a "colagem tectônica" entre os segmentos distintos da mesma placa litosférica poderá desencadear episódios de exumação mantélica intraplaca e surgimento consequente de faixa geotermal anômala. Neste caso, os segmentos continental e oceânico da placa Sul-Americana devem ser considerados como sub-placas com baixo grau de colagem ao longo da MCB.

É importante notar também as características na distribuição geográfica das jazidas de petróleo e gás na MCB. Estas se encontram localizadas na parte central da faixa geotermal, conforme ilustrada no mapa da Figura (6). Implica-se na existência de uma associação direta entre o campo geotérmico e zonas de reservatórios de hidrocarbonetos.

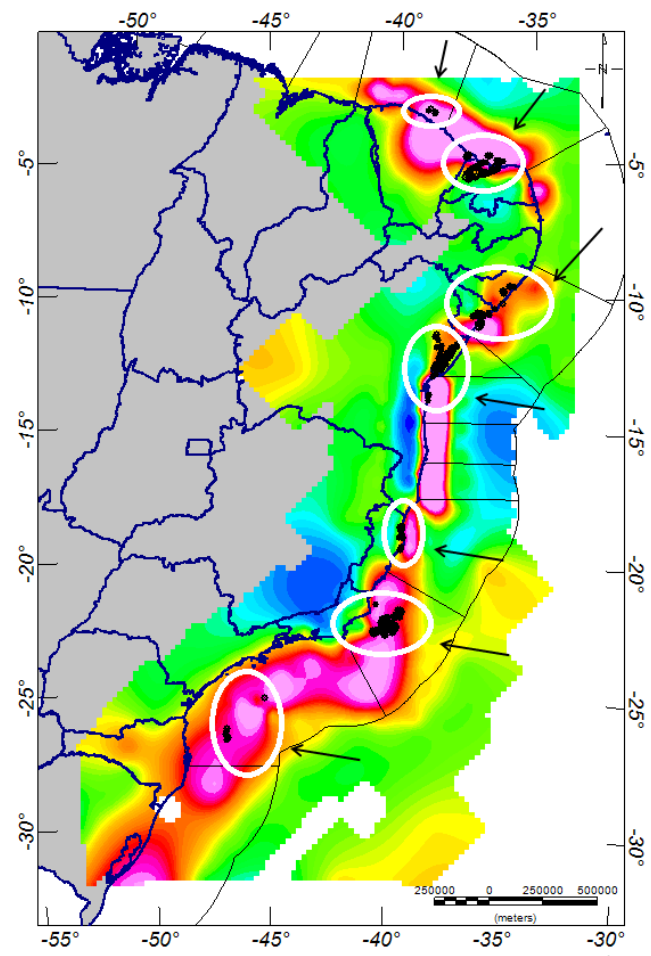

Figura 6 - Mapa indicando associação entre faixa geotermal anômala e jazidas de hidrocarbonetos ao longo da $M C B$ 


\section{Agradecimentos}

Este trabalho foi realizado como parte da Tese de Doutorado de trabalho do primeiro autor. Agradecemos Dr. Andreas Papa pelo apoio institucional. O segundo autor deste trabalho é recepiente de bolsa CNPq de Produtividade de Pesquisa (Projeto No. 301865/2008-6).

\section{Referências}

American Association of Petroleum Geologists (AAPG), 1976, Basic data file from AAPG Geothermal Survey of North America: Univ. of Oklahoma, Norman.

Andrade Fontes, L. C. A. 1980. Determinação de Fluxo Geotérmico na bacia sedimentar Sergipe-Alagoas. Unpublished M. Sc. Thesis, Univ. Federal da Bahia, Salvador.

Asmus, H. E., Ponte, F. C., 1973. The Brazilian Marginal Basins, In Nairn, A.E.M. and F.G. Stehili, eds, The Ocean Basins and Margins, v.1 - The South Atlantic, Plenum Press, Nova York, p.87-133.

Cainelli, C., Mohriak, W. U., 1999. Some remarks on the evolution of sedimentary basins along the eastern Brazilian continental margin, Episodes, 22(3):206-216.

Cardoso, R. A., Hamza, V. M., 2003. Gradiente e Fluxo Geotérmico da Plataforma Continental da Região Sudeste do Brasil. $8^{\text {th }}$ International Congress of the Brazilian Geophysical Society, Rio de Janeiro.

Cardoso, R. A., Hamza, V. M., 2014. Heat Flow in the Campos Sedimentary Basin and Thermal History of the Continental Margin of Southeast Brazil, ISRN Geophysics, 1-19, doi.org/10.1155/2014/384752.

Carvalho, H. S., 1981. Métodos para determinação de fluxo geotérmico com aplicação às bacias sedimentares petrolíferas do Recôncavo baiano (Brasil) e Sumatra (Indonésia), Tese de Doutorado, UFBA.

Del Rey, A. C., Zembruscki, S. G., 1991. Estudo hidrogeotérmico das bacias do Espírito Santo e Mucuri. Bol. Geoc. Petrobrás, RJ, 5 (1/4): 25-38.

Hamza, V. M., Verma, R. K., 1969. The relationship of heat flow with the age of basement rocks.. Bulletin Volcanologique, Napoli, Italia, v. 33, p. 123-152.

Houbolt J. J. H. C., Wells, P. R. A., 1980. Estimation of heat flow in oil wells based on a relation between heat condutivity and sound velocity. Geologie en Mijnbouw, 59(3), 215-24.

Matos, R. M. D., 2000. Tectonic evolution of the Equatorial South Atlantic. In Mohriak, W. U. and Talwani, M. Eds., Atlantic rifts and continental margins, pp. 331354. American Geophysical Union, Geophysical Monograph 115, Washington, USA.

Mascle, J., Blarez, E., Marinho, M., 1988. The shallow structures of the Guinea and Ivory Coast-Ghana transform margins: their bearing on the Equatorial Atlantic Mesozoic evolution. Tectonophysics, 188:193-209.

Milani, E. J., Thomaz F. A., 2000. Sedimentary basins of South America. In: Cordani, U.G.; Milani, E.J.; Thomaz Filho, A.; and Campos, D.A. (Eds.), Tectonic evolution of South America. Rio de Janeiro, $31^{\text {st }}$ International Geological Congress, pp. 389-452.

Ojeda, H. A. O., 1982. Structural Framework, Stratigraphy, and Evolution of Brazilian Marginal Basins: AAPG Bulletin, v.66, p.732-749.

Polyak, B. G. e Smirnov, Y. B. 1968. Relation between terrestrial heat flow and the tectonics of continents. Geotectonics, p. 205-213.

Ross, S., Pantoja, J. L., 1978. Estudo geotérmico da bacia de Campos, Relatório Interno, PETROBRÁs, DEPEX, DISUD, Vitória (ES).

Rossi F. J. 1981. Mapa de gradiente geotérmico na plataforma continental brasileira. Relatório Interno, CENPES/SUPEP/DIVEX/SEGEL, 24pp.

Szatmari, P., Françolin, J. B. L., Zanotto, O. A., Wolff, S., 1987. Evolução tectônica da margem equatorial brasileira. Revista Brasileira de Geociências 17, 180-188.

Viana, S. M. 1999, Fluxo Térmico em uma Bacia Sedimentar da Margem Continental Brasileira. Monografia de Graduação - FGEL/UERJ.

Vieira, F. P., Hamza, V. M., 2011. Global heat flow: comparative analysis based on experimental data and theoretical values. Proceedings of the 12th International Congress of the Brazilian Geophysical Society, Rio de Janeiro.

Zalán, P. V., 1985. Tectonics and sedimentation of the Piauí-Camocin sub-basin, Ceará Basin, offshore northeastern Brazil. Petrobras, Série Ciência-TécnicaPetróleo 17, Rio de Janeiro.

Zembruscki, S. G., 1982. Gradiente geotermico das bacias terrestres Brasileiras Geothermal gradients in Brazilian continental basins, Publ. Petroleo Brasileiro S.A., 57 pp.

Zembruscki, S. G., Kiang, C. H., 1989. Gradiente geotérmico das bacias sedimentares brasileiras. Bol. Geoc. Petrobrás, Rio de Janeiro, 3(3): 215-227. 